\title{
MicroRNA-22 enhances the differentiation of mouse induced pluripotent stem cells into alveolar epithelial type II cells
}

\author{
Fan Tao, ${ }^{1}$ Feng Wang, ${ }^{2}$ Weichen Zhang, ${ }^{1}$ Yaming $\mathrm{Hao}^{1}$ \\ ${ }^{1}$ Department of Traditional Chinese Medicine, Wuhan Fifth Hospital, Wuhan \\ ${ }^{2}$ School of Physical Education, Wuhan Business University, Wuhan, China
}

\begin{abstract}
Considerable evidence has verified that microRNAs (miRNAs) play important roles in various cellular processes including differentiation. However, the regulatory roles of miRNAs involved in the differentiation of induced pluripotent stem cells (iPSC) into lung epithelial cells are still unknown. In this study, we first evaluated the current protocols to differentiate iPSC into alveolar epithelial type II (AEC II) cells, but the efficiency is low. We next identified that miR-22 can efficiently enhance the differentiation of iPSC into AEC II cells under the stimulation of proper growth factors and growing on appropriate matrix. Moreover, the AEC II cells generated from iPSC with miR-22 overexpression can proliferate and secrete lung surfactant. Here, we discovered a previously unknown interaction between miR-22 and iPSC differentiation but also provide a potential target for the effective derivation of AEC II from iPSCs for cell-based therapy.
\end{abstract}

Key words: miR-22; iPSC; differentiation; AEC II; cell-based therapy.

Correspondence: Fan Tao, PhD, Department of Traditional Chinese Medicine, Wuhan Fifth Hospital, No. 122, Xianzheng Road, Hanyang District, Wuhan, China. E-mail: taofan421@126.com

Contribution: FT, FW, study concept, experiments design; FT, manuscript drafting; FW, WZ, experiments completion; WZ, YH, data analysis, manuscript revision.

FT and FW contribute equally to this study.

Conflict of interest: The authors declare that they have no competing interests.

Availability of data and materials: The datasets used and/or analyzed during the current study are available from the corresponding author on reasonable request.

Ethical approval: Ethical approval was obtained for all experimental procedures by the Ethical Committee of the Wuhan Fifth Hospital, Wuhan, China.

Patient consent for publication: Not applicable. 


\section{Introduction}

The alveolar epithelium plays an important role in remodeling, repair, and homeostasis of the lung under both physiological and pathological conditions and serves as a barrier against pathogens, allergens, and pollutants in the inspired air. ${ }^{1}$ Acute lung injuries (ALI) as well as chronic lung diseases, such as idiopathic pulmonary fibrosis (IPF) and chronic obstructive lung disease (COPD), constitute a significant health burden worldwide. ${ }^{2,3}$ Current medications can only reduce symptoms or delay disease progression. Therefore, cell-based therapies become an important new frontier in lung research and disease treatment due to the cells' ability to orchestrate physiological processes in response to local signaling cues. One possible cell source for cell-based treatment is induced pluripotent stem cells (iPSC). AEC II cells are usually cuboidal in shape and cover only about $7 \%$ of the total alveolar surface area. ${ }^{4}$ AEC II cells are responsible for epithelium reparation upon injury and ion transport. In addition, alveolar epithelial type II (AEC II) cells also contribute to lung defense by secreting lipoprotein material called surfactant, which is responsible for to reducing the surface tension in the alveoli. Moreover, AEC II cells can self-renew and transdifferentiate into alveolar epithelial type I (AEC I) cells. Thus, AEC II cells become an attractive candidate for cell therapies of lung diseases. MicroRNAs (miRNAs) play critical roles in various cellular processes including differentiation. ${ }^{5}$ MiR-130a has been found to control bone marrow mesenchymal stem cell differentiation towards the osteoblastic and adipogenic fate. ${ }^{6} \mathrm{MiR}-690$ also has been validated to regulate iPSCs differentiation into insulin-producing cells by targeting Sox $9 .^{7}$ Moreover, overexpression of miR-375 and miR-122 promotes the differentiation of human induced pluripotent stem cells into hepatocyte-like cells. ${ }^{8}$ However, it is still unclear through which miRNAs can promote the differentiation of iPSCs into AEC II cells.

In this study, we identified that miR-22 can promote the differentiation of iPSCs into AEC II cells. These iPSC-derived AEC II with miR-22 overexpression are capable to proliferate and secrete pulmonary surfactant. Our findings provide new insight to miR-22 functions and iPSCs differentiation, but also provide a potential target for the derivation of AEC II from iPSCs for cell-based therapy.

\section{Materials and Methods}

\section{Culture of mouse iPSCs}

The mouse iPSCs (iPS-MEF-Ng-492B-4) were purchased from Cell Bank (RIKEN BioResource Center, Japan) were propagated on the mitomycin C (Sigma-Aldrich, Shanghai, China) inactivated Mouse Embryo Fibroblast (MEF) cells (ATCC, Manassas, VA, USA), which can be used as feeder layer to condition the medium for iPSCs. Briefly, MEF cells were cultured in Dulbecco's Modified Eagle's Medium (DMEM) (Sigma-Aldrich, Shanghai, China) supplemented by $10 \%$ fetal bovine serum (FBS) (TBD Biotechnology Development, Tianjin, China), $50 \mathrm{U} / \mathrm{mL}$ penicillin and $50 \mathrm{mg} / \mathrm{mL}$ streptomycin (Sigma-Aldrich, Shanghai, China). After the cell division was halted with Mitomycin C (Sigma-Aldrich, St. Louis, MO), a single MEF cell suspension $\left(5 \times 10^{5}\right.$ cells) was seeded on a $0.1 \%$ gelatin (Sigma-Aldrich, St. Louis, MO, USA) coated $25 \mathrm{~cm}^{2}$ flask. The next day, MEF medium was removed and $5 \mathrm{~mL}$ of mouse iPSC medium consisted of DMEM (high glucose, without sodium pyruvate) (Sigma-Aldrich, Shanghai, China), 15\% FBS, $0.1 \mathrm{mM}$ nonessential amino acids (Sigma-Aldrich, Shanghai, China ), 0.1 mM 2-mercaptoethanol (Sigma-Aldrich, Shanghai, China), 1000 $\mathrm{U} / \mathrm{mL}$ mouse LIF (Millipore, Billerica, MA, USA), $50 \mathrm{U} / \mathrm{mL}$ penicillin, and $50 \mathrm{mg} / \mathrm{mL}$ streptomycin was added.

\section{In vitro differentiation of iPSCs into AEC II cells}

Pure iPSCs were used to drive AEC II cells after removal of MEF cells. Cells were first trypsinized, centrifuged, resuspended in iPSCs medium, and plated to a $25 \mathrm{~cm}^{2}$ flask without coated gelatin. The cells were incubated at $37^{\circ} \mathrm{C}$ with $5 \% \mathrm{CO}_{2}$ for $1 \mathrm{~h}$. Most MEF cells were attached to the bottom of the flask. The supernatant containing iPSCs was collected and centrifuged at $200 \mathrm{~g}$ for $5 \mathrm{~min}$ to get the purified iPSCs. Then, we used a two-step differentiation protocol to drive iPSCs into AEC II cells as previous described. ${ }^{9}$ The first step was endoderm induction in the differentiation medium with Activin A (20 ng/mL, Sigma-Aldrich, Shanghai, China) for 6 days. The differentiation medium consisted of 25\% (v/v) Ham's F12 medium (Sigma-Aldrich, Shanghai, China), 75\% (v/v) Iscove's modified Dulbecco's medium (Sigma-Aldrich, Shanghai, China) supplemented with $0.5 \mathrm{x}$ of N-2 Supplement and B-27 Supplement minus vitamin A (Invitrogen, Beijing, China), 0.05\% BSA, 2 mM glutamine (Invitrogen, Beijing, China), $0.5 \mathrm{mM}$ ascorbic acid (Sigma-Aldrich, Shanghai, China), $0.45 \mathrm{mM}$ 1-thioglycerol (Sigma-Aldrich, Shanghai, China), $50 \mathrm{U} / \mathrm{mL}$ penicillin and $50 \mathrm{mg} / \mathrm{ml}$ streptomycin. The second step was lung epithelial induction in the differentiation medium containing FGF-2 (50 ng/mL, Sigma-Aldrich, Shanghai, China) for 5 days.

\section{Immunocytochemistry}

Immunostaining was carried out to quantify iPSCs. The purified cells were seeded onto a gelatin-coated 24-well cell tissue culture plate $\left(1 \times 10^{6}\right.$ cells/well $)$. On the next day, cells were washed with phosphate-buffered saline (PBS, Solarbio Science \& Technology Co., Ltd., Beijing, China), fixed with $4 \%$ paraformaldehyde in PBS for $30 \mathrm{~min}$ at room temperature, and permeabilized with $0.1 \%$ Triton X-100 (CWBIO, Beijing, China) for $15 \mathrm{~min}$. The fixed cells were blocked with $1 \%$ bovine serum albumin (Beijing Solarbio Science \& Technology Co.) for $1 \mathrm{~h}$ and were then incubated with mouse monoclonal anti-Nanog (Cell Signaling Technology, Beverly, MA, USA) or anti-SSEA-1 (Cell Signaling Technology) at $4^{\circ} \mathrm{C}$ overnight. The cells were washed and incubated with Alexa-Fluor-488-conjugated anti-(mouse IgG) secondary antibodies (Cell Signaling Technology). For the AEC II cell marker, cells were incubated with mouse monoclonal mouse anti-SP-C (Santa Cruz Biotechnology, Santa Cruz, CA, USA) at $4{ }^{\circ} \mathrm{C}$ overnight. The next day, cells were washed and incubated with Alexa-Fluor-546-conjugated anti- (mouse IgG) secondary antibodies (Cell Signaling Technology).

The 4',6-Diamidino-2-phenylindole dihydrochloride (DAPI) $(2.5 \mathrm{mg} / \mathrm{mL})$ (Sigma-Aldrich, Shanghai, China) was used as nuclear staining.

\section{RNA isolation and quantitative real-time PCR (qRT-PCR)}

Total RNAs were extracted using RNeasy mini kit (Qiagen, Beijing, China). Concentration and RNA purity were estimated by a NanoDrop ND1000 spectrophotometer (NanoDrop Technologies, Rockland, DE, USA). First-strand cDNA was synthesized by reverse transcription with first strand cDNA synthesis kit (Roche, Basel Switzerland). The specific gene expression was determined by Taqman qRT-PCR and performed on a QuantStudio 6 Flex system (Thermofisher, Gaithersburg, MD, USA). $\beta$-Actin was used as an internal control.

To quantify the mature miRNAs, total RNA was reverse transcribed using the Taqman advanced miRNA cDNA synthesis kit (Applied Biosystems, Bedford, MA, USA) according to manufacturer recommended protocols. For miRNA expression normalization, U6 small nuclear RNA (snRNA) was used as the reference control and reverse transcribed by TaqMan microRNA reverse transcription kit following manufacturer's protocol (Applied Biosystem, Bedford, MA, USA).

All the specific primers for genes and miRNA expression are 
commercially available from Applied Biosystem. The fold change of gene or miRNA transcript levels between different samples was calculated with the equation $2-\Delta \Delta \mathrm{Ct}^{10}$.

\section{miRNA overexpression}

The lentiviral miR-22 expression vector was constructed as previous study. ${ }^{11}$. Lentiviruses were generated by co-transfecting $0.9 \mu \mathrm{g}$ of lentiviral vector [premiR-22 or empty vectors (VC); System Biosciences, Mountain View, CA, USA] and $2.7 \mu \mathrm{g}$ of Lenti-X HTX Packaging Mix (Clontech, Mountain View, CA, USA) in 293T cells. Supernatants were collected $48 \mathrm{~h}$ after transfection, filtered through a $0.45-\mu \mathrm{m}$ membrane, tittered, and stored at $-80^{\circ} \mathrm{C}$ until use.

\section{Isolation of mouse primary AEC II}

Mouse AEC II were isolated from 8-week male C57BL/6 mice (SLAC Laboratory Animals Company Limited, Shanghai, China) based on the previously reported procedure ${ }^{12,13}$ with modification. Mice were anesthetized and lungs were perfused with solution (10 $\mathrm{mM}$ HEPES, pH 7.4, 0.9\% NaCl, 0.1\% glucose, $5 \mathrm{mM} \mathrm{KCl}, 1.3$ $\mathrm{mM} \mathrm{MgSO} 4,1.7 \mathrm{mM} \mathrm{CaCl} 2,0.1 \mathrm{mg} / \mathrm{mL}$ streptomycin sulfate, $0.06 \mathrm{mg} / \mathrm{mL}$ penicillin $\mathrm{G}, 3 \mathrm{mM} \mathrm{Na} 2 \mathrm{HPO} 4$ and $3 \mathrm{mM} \mathrm{NaH} 2 \mathrm{PO} 4$, Sigma-Aldrich, Shanghai, China), followed by instilling $1 \mathrm{~mL}$ of above solution with dispase (500 caseinolytic units/mL; SigmaAldrich, Shanghai, China) directly through the trachea. The isolated lungs were pooled into a beaker containing the solution with dispase and incubated at $37^{\circ} \mathrm{C}$ for $45 \mathrm{~min}$. After then, the lungs were chopped into small pieces and further digested with the addition of DNase I (100 $\mu \mathrm{g} / \mathrm{mL}$, Sigma-Aldrich, Shanghai, China) with shaking at $37^{\circ} \mathrm{C}$ for $45 \mathrm{~min}$. Subsequently, the digested lungs were sequentially filtered through 160-, 37- and 15- $\mu \mathrm{m}$ gauge nylon mesh. The filtrate was centrifuged at $250 \mathrm{~g}$ for $10 \mathrm{~min}$. The cell pellet was resuspended in DMEM and incubated in a $100-\mathrm{mm}-$ diameter Petri dish coated with mouse $\operatorname{IgG}(75 \mu \mathrm{g} / \mathrm{dish})$ for 1 hour. The cells were spun down at $250 \mathrm{~g}$ for $10 \mathrm{~min}$ and resuspended in
DMEM with $10 \%$ FBS. The freshly isolated primary AEC II were used as a positive control for the subsequent proliferation and ELISA assay.

\section{Proliferation assay}

5-bromo-2- deoxyuridine (BrdU) cell proliferation assay kit (Millipore, Billerica, MA, USA) was carried out to detect cell proliferation of freshly isolated AEC II and AEC II differentiated from iPSCs by the manufacturer's protocol.

\section{ELISA for SPC}

ELISA was performed on cell culture medium collected before and after stimulation of iPSC-AEC II or freshly isolated primary AEC II to quantify secreted SP-C using SP-C ELISA kit (Lsbio, Seattle, WA USA) following the manufacturer's instructions. Freshly isolated primary AEC II and AEC II derived from iPSCs were cultured in 96-well plates overnight. Cells were then washed twice by PBS and DMEM with 10\% FBS was added. The cells were incubated at $37^{\circ} \mathrm{C}$ for $30 \mathrm{~min}$. Culture medium was removed as a zero-time control $(0 \mathrm{~h})$ for one set of wells. After then, cells were stimulated with lung surfactant secretagogues $(100 \mu \mathrm{M}$ ATP, $0.1 \mu \mathrm{M}$ PMA and $10 \mu \mathrm{M}$ terbutaline; ATP+PMA+Terb, SigmaAldrich, Shanghai, China) for $2 \mathrm{~h}$ at $37^{\circ} \mathrm{C}$. Unstimulated cells in parallel cultures were used as controls.

\section{Statistics}

Results are shown as means $\pm \mathrm{SE}$ (standard errors). Unpaired Student's $t$-test for two groups or one-way analysis of variance (ANOVA) test, followed by Tukey's multiple comparison for multiple groups were used to compare the significance of differences between the mean of different groups. SPSS (SPSS Inc. Chicago, IL, USA) was used to calculate the significance. A p value less than 0.05 is statistically significant.

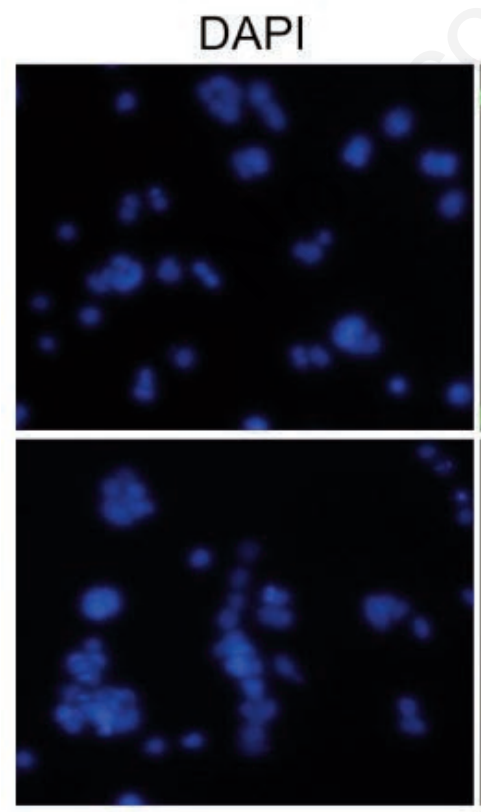

DAPI

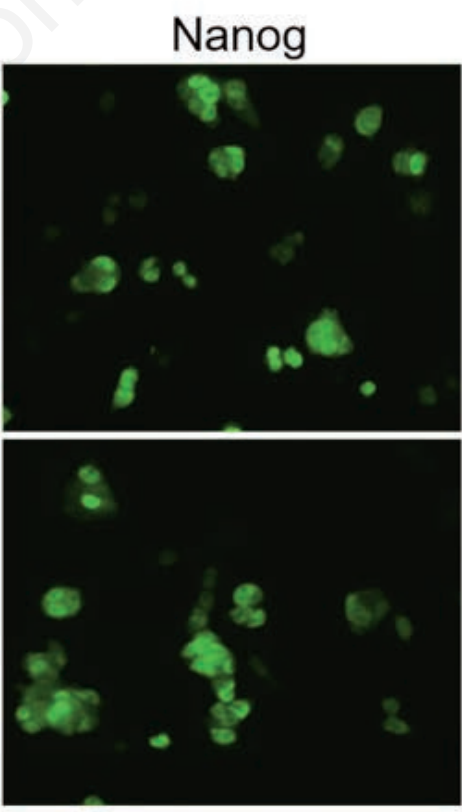

SSEA-4

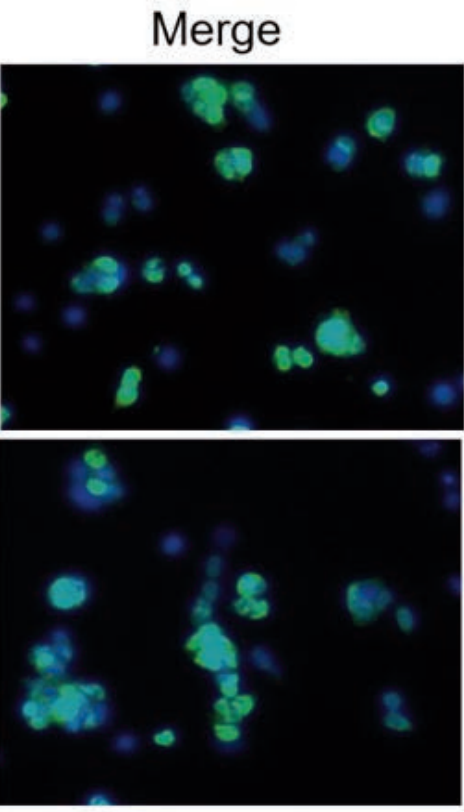

Merge

Figure 1. Identification of mouse iPSCs by immunostaining after removal of MEF. Representative images (20x) for pluripotent markers, Nanog and SSEA-4, expression in the iPSC colonies. DAPI staining was used as a nuclear marker. 


\section{Results}

\section{Purity of iPSCs}

Immunostaining and qRT- PCR were used to determine the characterization of iPSCs. As shown in Figure 1, immunostaining confirmed that iPSCs expressed the pluripotency markers NANOG and SSEA-4. There were around $95 \pm 1.34 \%$ and $96 \pm 0.34 \%$ Nanog and SSEA-4 positive cells after removal of MEF (Figure 1). In our studies, only iPSCs with more than $95 \%$ purity were used for the subsequent studies.

\section{Differentiation of iPSCs to AEC II cells}

Mouse iPSCs were induced to differentiate into functional AEC II cells using the protocol developed previously. ${ }^{9}$ Purified iPSCs were seeded on collagen IV coated 6-well plates for $24 \mathrm{~h}$. Next, the iPSC medium was switched to the differentiation medium with or without Activin A for 6 days, and then with or without FGF-2 for another 5 days. As shown in Figure 2A, the levels of SP-

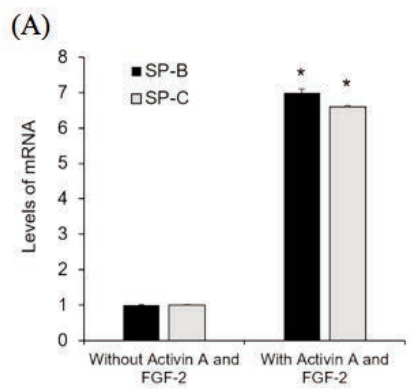

(B)
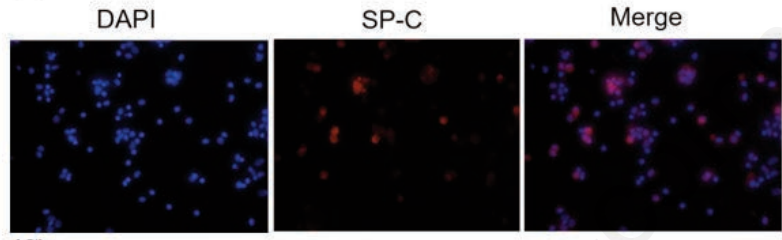

(C)

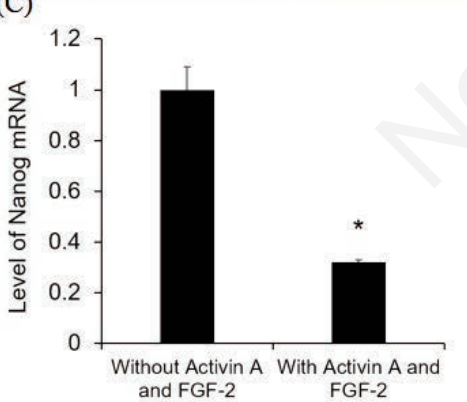

Figure 2. Characterization of differentiated cells from iPSCs. A) Levels of SP-B and SP-C mRNA increase in differentiated iPSC cells as determined by qRT-PCR; black bar: iPSCs cultured in differentiation medium without Activin A and FGF-2 for 11 days; gray bar: iPSCs cultured with Activin for 6 days and FGF-2 for the subsequent 5 days; ${ }^{*} \mathrm{P}<\mathbf{0 . 0 5} v s$ without Activin and FGF-2 $(n=3)$. B) Immunostaining analysis of AECII cell markers of SPC (20x). C) level of Nanog mRNA reduces in differentiated iPSC cells as determined by $q R T-P C R$; black bar: iPSCs cultured in differentiation medium without Activin A and FGF-2 for 11 days; gray bar: iPSCs cultured with Activin for 6 days and FGF-2 for the subsequent 5 days; ${ }^{*} \mathrm{P}<0.05$ vs without Activin and FGF-2 $(\mathbf{n}=3)$.
$\mathrm{B}$ and SP-C mRNA were significantly enhanced after adding activin $\mathrm{A}$ and FGF-2 compared with the differentiation medium without activin A and FGF-2. Moreover, immunostaining analysis also confirmed that $37.21 \pm 5 \%$ of the differentiated iPSCs are positive for SP-C which is one of the AEC II cell markers (Figure 2B). In addition, we also monitored the level of Nanog mRNA in the differentiated iPSCs with Activin A and FGF as well. We found that the Nanog mRNA levels were strongly decreased in comparison with cells cultured in differentiation medium without activin A and FGF-2 (Figure. 2C). These data indicated that Activin A and FGF can induce the differentiation of AEC II cells from iPSCs, but the efficiency is low.

\section{MiR-22 efficiently enhanced the differentiation of iPSCs to AEC II in vitro}

In our preliminary data, miR-22 was highly expressed in adult lungs compared to fetal lung. Since the differentiation of AEC II occurs at the late stages of fetal lung development and continues until adult, miR-22 overexpression may enhance the differentiation of iPSCs into AECs. So, we subsequently determined whether
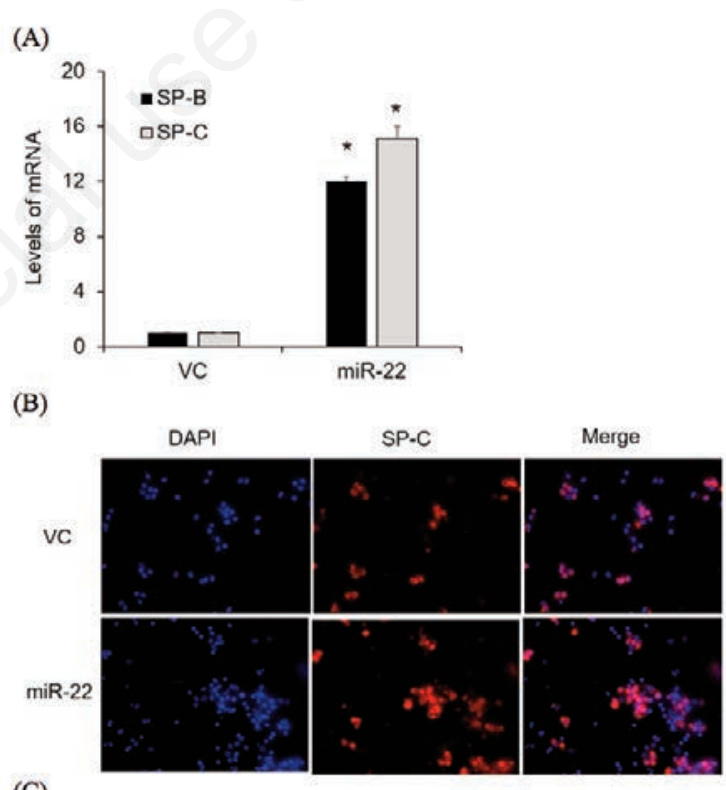

(C)

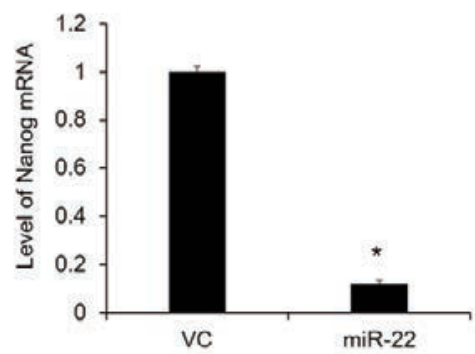

Figure 3. Verification of differentiated cells from iPSCs with miR22 overexpression. A) Levels of SP-B and SP-C mRNA increase in miR-22 overexpressed cells compared with $\mathrm{VC}$; ${ }^{*} \mathrm{P}<0.05$ vs $\mathrm{VC}$ $(\mathbf{n}=3)$. B) Immunostaining analysis of AECII cell markers of SPC in miR-22 or VC overexpressed cells (20x). C) level of Nanog mRNA significantly decreases in cells with miR-22 overexpression than VC as determined by $\mathrm{qRT}-\mathrm{PCR} ;{ }^{*} \mathbf{P}<0.05$ vs VC $(\mathbf{n}=3)$. 
miR-22 can enhance the differentiation efficiency of iPSCs into AEC II cells.

iPSCs were seeded on collagen IV-coated plates and infected with a lentiviral virus expressing miR-22 or control vector (VC). After $24 \mathrm{~h}$, the cells were serum-starved overnight and then cultured in differentiation medium with Activin A for 6 days and then FGF-2 for 5 days (11 days). As shown in Figure 3A, miR-22 significantly enhanced the expression of SP-B and SP-C mRNA compared with VC by qRT-PCR for immunostaining, the SP-C-positive cells were $49.12 \%$ in cells with miR-22 overexpression as compared to $35.34 \%$ in VC (Figure 3B). The levels of mRNA expression of iPSC marker, Nanog, were also detected. We found the level of Nanog mRNA was remarkably decreased in the cells with miR-22 overexpression compared to VC (Figure. 3C).

\section{iPSC derived AEC II are functional}

To decipher whether the differentiated ACE II cells are functional or not, we next investigated the proliferation of the iPSCs derived ACE II with miR-22 (iPSC-AEC II-miR-22) and VC (iPSC-AEC II-VC) by BrdU assay in vitro. We found that iPSC-

(A)

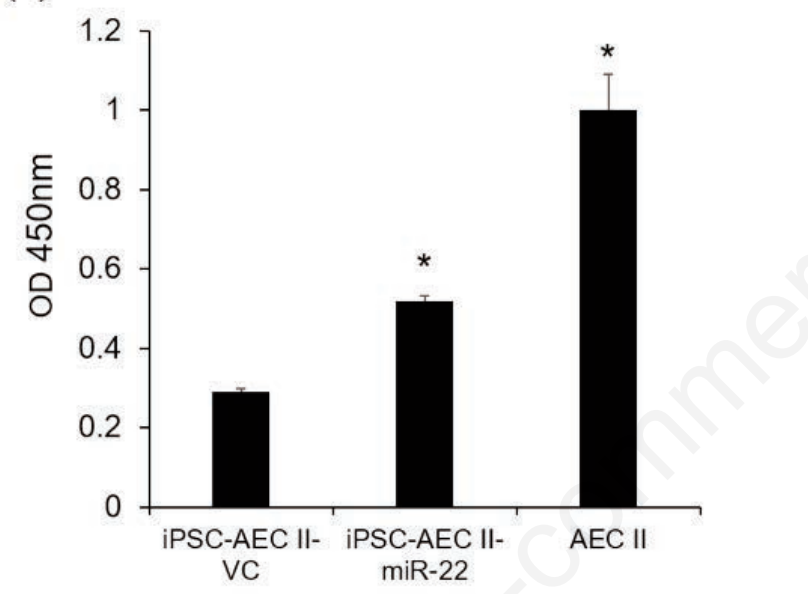

(B)

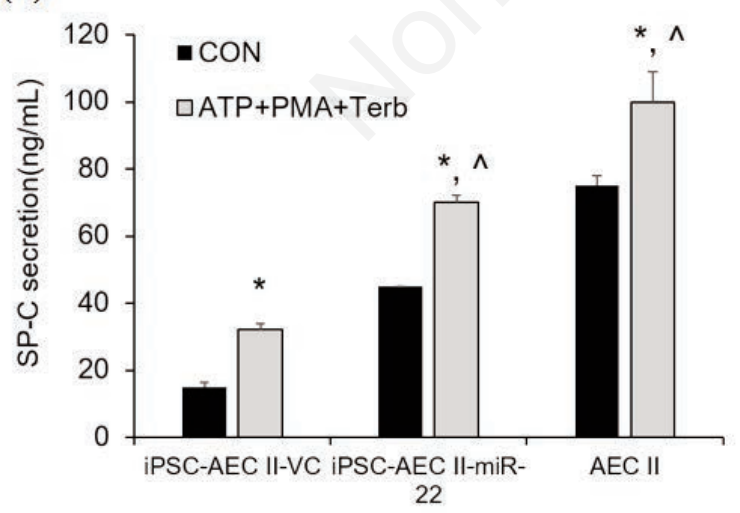

Figure 4. iPSC- AEC II-miR-22 cells proliferate and secret SPC. A) miR-22 strongly enhanced the proliferation compared with VC; primary AEC II cells used as positive controls; ${ }^{*} \mathrm{P}<\mathbf{0 . 0 5}$ vs VC (n=3). B) Primary AEC II cells, iPSC- AEC II-miR-22 cells and iPSC-AEC II-VC cells were stimulated for $2 \mathrm{~h}$ with lung surfactant secretagogues (ATP+PMA+Terb); SP-C secretion were measured; Con: without with lung surfactant secretagogues; ${ }^{*} \mathrm{P}<0.05$ vs Con $(\mathrm{n}=3) ;{ }^{\wedge} \mathrm{P}<\mathbf{0 . 0 5}$ vs VC $(\mathrm{n}=3)$.
AEC II-miR-22 cells strongly enhanced the proliferation compared with iPSC-AEC II-VC, although less compared to mouse primary AEC II cells (Figure 4A).

One of the major functions of AEC II is to secrete lung surfac$\tan t^{4}$. So, we subsequently examined whether iPSC derived AEC II can secrete lung surfactant protein. As shown in Figure 4B, the SP$\mathrm{C}$ secretion of iPSC-AEC II-miR-22 was significantly increased compared with iPSC-AEC II-VC after lung surfactant secretagogues (ATP, PMA and terbutaline) stimulation. Primary AEC II was used as positive control (Figure 4B). These results suggest that iPSC-AEC II-miR-22 behave similarly to the freshly isolated mouse AEC II in vitro.

\section{Discussion}

Alveolar epithelial injury is a hallmark of a variety of acute and chronic lung diseases. Present medications can only stabilize the disease conditions or delay disease progression. Although lung transplantation is the final treatment option for these advancedstage lung diseases, donor organ shortage is still a major problem. Therefore, the stem cell therapy provides a new strategy for repairing severe acute and chronic lung injuries.

Numerous studies have demonstrated the therapeutic potential of iPSCs. For instance, it has been reported that a long-lasting, but finite AEC II cell line can be derived from iPSC as a novel cellular model to study alveolar epithelial cell biology in lung health and disease. ${ }^{14}$ AEC II become an attractive candidate for cell therapies of lung diseases since these cells have recently been identified as alveolar stem cells, ${ }^{15}$ and secrete a high level of lung surfactant in the distal alveoli. Human iPSCs have also been verified to derive lung progenitor and alveolar epithelial cells and can attenuate hyperoxia-induced lung injury. ${ }^{16}$ However, it is still a challenge to generate large quantities of pure AEC II. The conditions for directing iPSCs to differentiate into an alveolar epithelial lineage with homogeneity are not fully defined yet.

miRNA causes a fast response in protein expression based on inhibition of mRNA translation and stability. Their ability to regulate different proteins can rapidly impose a dominant phenotypic change in cell destination. It has been reported that miRNAs can affect the differentiation of iPSCs. Inhibition of microRNA-495 has been found to enhance therapeutic angiogenesis of human iPSCs. ${ }^{17}$ MiR-140 has also been validated to enhance chondrogenesis differentiation of human iPSCs with transforming growth factor beta 3 (TGF $\beta 3) .{ }^{18}$

In this study, we used a two steps method to differentiate AEC II from mouse iPSCs and found that miR-22 can effectively enhance the differentiation of mouse iPSCs into AEC II. Moreover, these iPSC-AEC II with miR-22 overexpression are capable of proliferating and secreting lung surfactant. However, it is still worthy verifying the downstream targets and signaling pathways of miR-22, and considering the in vivo study in the future. Our studies represent an initial and important step towards the ultimate goal of cell-based therapy of lung injuries.

\section{References}

1. Fehrenbach H. Alveolar epithelial type II cell: defender of the alveolus revisited. Respir Res 2001;2:33-46.

2. Barnes PJ. Cellular and molecular mechanisms of chronic obstructive pulmonary disease. Clin Chest Med 2014;35:71-86.

3. Bhattacharya J, Matthay MA. Regulation and repair of the alveolar-capillary barrier in acute lung injury. Annu Rev 
Physio. 2013;75:593-615.

4. Chuquimia OD, Petursdottir DH, Periolo N, Fernandez C. Alveolar epithelial cells are critical in protection of the respiratory tract by secretion of factors able to modulate the activity of pulmonary macrophages and directly control bacterial growth. Infect Immun 2013;81:381-9.

5. Li N, Long B, Han W, Yuan S, Wang K. microRNAs: important regulators of stem cells. Stem Cell Res Ther 2017;8:110.

6. Lin Z, He H, Wang M, Liang J. MicroRNA-130a controls bone marrow mesenchymal stem cell differentiation towards the osteoblastic and adipogenic fate. Cell Prolif 2019;52:e12688.

7. $\mathrm{Xu} \mathrm{Y,} \mathrm{Huang} \mathrm{Y,} \mathrm{Guo} \mathrm{Y,} \mathrm{Xiong} \mathrm{Y,} \mathrm{Zhu} \mathrm{S,} \mathrm{Xu} \mathrm{L,} \mathrm{et} \mathrm{al.}$ microRNA-690 regulates induced pluripotent stem cells (iPSCs) differentiation into insulin-producing cells by targeting Sox9. Stem Cell Res Ther 2019;10:59.

8. Jaafarpour Z, Soleimani M, Hosseinkhani S, Geramizadeh B, Yaghmaei P, Mobarra N, Karimi MH. Overexpression of microRNA-375 and microRNA-122 promotes the differentiation of human induced pluripotent stem cells into hepatocytelike cells. Biologicals 2020;63:24-32.

9. Roszell B, Mondrinos MJ, Seaton A, Simons DM, Koutzaki $\mathrm{SH}$, Fong GH, et al. Efficient derivation of alveolar type II cells from embryonic stem cells for in vivo application. Tissue Eng A 2009;15:3351-65.

10. Fu H, Tan J, Yin Q. Effects of recombinant adeno-associated virus-mediated CD151 gene transfer on the expression of rat vascular endothelial growth factor in ischemic myocardium. Exp Ther Med 2015;9:187-90.

11. Zhao C, Huang C, Weng T, Xiao X, Ma H, Liu L. Computational prediction of MicroRNAs targeting GABA receptors and experimental verification of miR-181, miR-216 and miR-203 targets in GABA-A receptor. BMC Res Notes 2012;5:91

12. Liu F, Zheng S, Liu T, Liu Q, Liang M, Li X, et al. MicroRNA21 promotes the proliferation and inhibits apoptosis in Eca109 via activating ERK1/2/MAPK pathway. Mol Cell Biochem 2013;381:115-25.

13. Wang J, Xia Y. Assessing developmental roles of MKK4 and MKK7 in vitro. Commun Integr Biol 2012;5:319-24.

14. Tamo L, Hibaoui Y, Kallol S, Alves MP, Albrecht C, Hostettler $\mathrm{KE}$, et al. Generation of an alveolar epithelial type II cell line from induced pluripotent stem cells. Am J Physiol Lung Cell Mol Physiol 2018;315:L921-32.

15. Barkauskas CE, Cronce MJ, Rackley CR, Bowie EJ, Keene DR, Stripp BR, et al. Type 2 alveolar cells are stem cells in adult lung. J Clin Invest 2013;123:3025-36.

16. Shafa M, Ionescu LI, Vadivel A, Collins JJP, Xu L, Zhong S, et al. Human induced pluripotent stem cell-derived lung progenitor and alveolar epithelial cells attenuate hyperoxia-induced lung injury. Cytotherapy 2018;20:108-25.

17. Liang J, Huang W, Cai W, Wang L, Guo L, Paul C, et al. Inhibition of microRNA-495 enhances therapeutic angiogenesis of human induced pluripotent stem cells. Stem Cells 2017;35:337-50.

18. Mahboudi H, Soleimani M, Enderami SE, Kehtari M, Ardeshirylajimi A, Eftekhary M, Kazemi B. Enhanced chondrogenesis differentiation of human induced pluripotent stem cells by MicroRNA-140 and transforming growth factor beta 3 ((TGFß3).). Biologicals 2018;52:30-36.

Received for publication: 4 August 2020. Accepted for publication: 1 September 2020.

This work is licensed under a Creative Commons Attribution-NonCommercial 4.0 International License (CC BY-NC 4.0).

(C) Copyright: the Author(s), 2020

Licensee PAGEPress, Italy

European Journal of Histochemistry 2020; 64(s2):3170

doi:10.4081/ejh.2020.3170 